CARPATHIAN J. MATH.

Volume 37 (2021), No. 1,

Pages $65-79$

\title{
Perturbed approximations of fixed points of nonexpansive mappings in $\mathrm{CAT}_{\mathrm{p}}(0)$ spaces
}

\author{
K. CALderón ${ }^{1}$, M. A. KhAmsi ${ }^{2}$ and J. MARtíneZ-Moreno ${ }^{3}$
}

ABSTRACT. Using the concept of the $\operatorname{CAT}_{\mathrm{p}}(0)$ spaces proposed by Khamsi et al. [Khamsi, M. A. and Shukri, S. A., Generalized CAT(0) spaces. Bull. Belg. Math. Soc. Simon Stevin, 24 (2017), No. 3, 417-426], we establish $\Delta$-convergence and strong convergence of a perturbed variant of Agarwal et al. $S$-iteration process for nonexpansive mapping. Finally, from them we deduce results valid for $\alpha$-nonexpansive mappings.

\section{INTRODUCTION}

In this section, we introduce the basic notations and terminologies which we will use throughout this work. A metric space $(X, d)$ is said to be a length space if any two points of $X$ can be joined by a rectifiable path (that is, a path of finite length) where the distance between any two points of $X$ is taken to be the infimum of the lengths of all rectifiable paths joining them. In this case, $d$ is said to be a length metric (otherwise known as an inner metric or intrinsic metric). In case no rectifiable path joins two points of the space, the distance between them is taken to be $\infty$.

A geodesic path joining $x \in X$ to $y \in X$ (or, more briefly, a geodesic from $x$ to $y$ ) is a map $c$ from closed interval $[0, l] \subset \mathbb{R}$ to $X$ such that $c(0)=x, c(l)=y$, and $d\left(c(t), c\left(t^{\prime}\right)\right)=$ $\left|t-t^{\prime}\right|$ for all $t, t^{\prime} \in[0, l]$. In particular, $c$ is an isometry and $d(x, y)=l$. The image $\gamma$ of $c$ is called a geodesic (or metric) segment joining $x$ and $y$. The space $(X, d)$ is said to be a geodesic space if every two points of $X$ are joined by a geodesic, and is said to be uniquely geodesic if there is exactly one geodesic joining $x$ and $y$ for each $x, y \in X$, which we denote by $[x, y]$, called the segment joining $x$ to $y$.

The most fundamental examples of geodesic metric spaces are normed vector spaces, As nonlinear examples, one can consider the $C A T(0)$ spaces [6].

A geodesic triangle $\Delta\left(x_{1}, x_{2}, x_{3}\right)$ in a geodesic metric space $(X, d)$ consists of three points in $X$ (called vertices of $\Delta$ ) and a geodesic segment between each pair of vertices (the edges of $\Delta)$. A comparison triangle for geodesic triangle $\Delta\left(x_{1}, x_{2}, x_{3}\right)$ in $(X, d)$ is a triangle $\bar{\Delta}\left(x_{1}, x_{2}, x_{3}\right):=\Delta\left(\bar{x}_{1}, \bar{x}_{2}, \bar{x}_{3}\right)$ in $\mathbb{R}^{2}$ such that $d_{\mathbb{R}^{2}}\left(\bar{x}_{i}, \bar{x}_{j}\right)=d\left(x_{i}, x_{j}\right)$ for $i, j \in\{1,2,3\}$. Such a triangle always exists [6].

Let $\Delta$ be a geodesic triangle in $X$ and $\bar{\Delta}$ its comparison triangle in $\mathbb{R}^{2}$. Then $\Delta$ is said to satisfy $C A T(0)$ inequality if for all $x, y \in \Delta$ and all comparison points $\bar{x}, \bar{y} \in \bar{\Delta}$, $d(x, y) \leq d_{\mathbb{R}^{2}}(x, y)$.

A geodesic metric space $X$ is called a $C A T(0)$ space if all geodesic triangles satisfy the above comparison axiom (i.e. $C A T(0)$ inequality). Some well known examples of $C A T(0)$

Received: 14.04.2020. In revised form: 20.01.2021. Accepted: 20.01.2021

2010 Mathematics Subject Classification. Primary 47H09, 47H10.

Key words and phrases. Fixed point, generalized $\operatorname{CAT}_{\mathrm{p}}(0)$ spaces, Hadamard metric spaces, hyperbolic metric spaces, modified Mann iteration, nonexpansive mapping, SM-iteration.

Corresponding author: J. Martínez-Moreno; jmmoreno@ujaen.es 
spaces are complete and simply connected Riemannian manifold having non-positive sectional curvature, pre-Hilbert spaces, R-trees, Euclidean buildings and the complex Hilbert ball with a hyperbolic metric.

Complete CAT(0) spaces are often called Hadamard spaces.

Definition 1.1. [19] A sequence $\left\{x_{n}\right\}$ in $X$ is said to $\Delta$-converge to $x \in X$ if $x$ is the unique asymptotic center of $\left\{u_{n}\right\}$ for every subsequence $\left\{u_{n}\right\}$ of $\left\{x_{n}\right\}$. In this case we write $\Delta-\lim _{n \rightarrow \infty} x_{n}=x$ and we call $x$ the $\Delta-\lim _{n \rightarrow \infty} x_{n}=x$.

Let $T: K \rightarrow K$ be a mapping. The set of fixed points of $T$ is denoted by $F(T)$. A mapping $T$ is said to be quasi-nonexpansive if $F(T) \neq \emptyset$ and $d(T x, p) \leq d(x, p)$ for all $x \in K$ and $p \in F(T) ; T$ is said to be nonexpansive if $d(T x, T y) \leq d(x, y)$ for all $x, y \in K$.

Lemma 1.1. [19]

i. Every bounded sequence in $X$ has $\Delta$-convergence subsequence.

ii. If $K$ is a closed convex subset of $X$ and if $\left\{x_{n}\right\}$ is a bounded sequence in $K$, then the asymptotic center of $\left\{x_{n}\right\}$ is in $K$.

iii. If $K$ is a closed convex subset of $X$ and if $f: K \rightarrow X$ is a nonexpansive mapping, then the conditions, $\left\{x_{n}\right\} \Delta$-converges to $x$ and $d\left(x_{n}, f\left(x_{n}\right)\right) \rightarrow 0$, imply $x \in K$ and $f(x)=x$

Naor and Silberman [27] extended the notion of $p$-uniformly convexity to the setting of geodesic spaces in the following way.

Definition 1.2. Fix $1<p<\infty$. A metric space $(X, d)$ is called $p$-uniformly convex with parameter $c>0$ iff $(X, d)$ is a geodesic space and for any three points $x, y, z \in X$ and all $t \in[0,1]$,

$$
d^{p}((1-t) x \oplus t y, z) \leq(1-t) d^{p}(x, z)+t d^{p}(y, z)-\frac{c}{2} t(1-t) d^{p}(x, y) .
$$

Note that inequality (1.1) guarantees that the space $X$ is uniquely geodesic (see [28, Lemma 2.2] for a proof in the case $p=2$ ). Also, any closed convex subset of a $p$-uniformly convex space is again a $p$-uniformly convex space with the same parameter.

Moreover, $C A T(0)$ spaces are 2-uniformly convex metric spaces with parameter $c=2$ and $C A T(\kappa)$ spaces $(\kappa>0)$ with $\operatorname{diam}(X)<\frac{\pi}{2 \sqrt{k}}$ are 2-uniformly convex metric spaces with parameter $c=(\pi-2 \sqrt{k} \epsilon) \tan (\sqrt{k} \epsilon)$ for any $\epsilon \leq \frac{\pi}{2 \sqrt{k}}-\operatorname{diam}(X)$ (see [2]).

It is easy to see that in a complete $p$-uniformly convex space, any bounded sequence has a $\Delta$-convergent subsequence. In the setting of $\mathrm{CAT}(0)$ spaces, $\Delta$-convergence is equivalent to another concept of weak convergence that uses projections on geodesic segments (see [12]). Reasoning as in [12], one can see that this equivalence also holds for $p$-uniformly convex spaces.

Definition 1.3. [20] Let $(X, d)$ be a geodesic metric space. $X$ is said to be a $\operatorname{CAT}_{\mathrm{p}}(0)$ space, for $p>2$, if for any geodesic triangle $\triangle$ in $X$, there exists a comparison triangle $\triangle$ in $l_{p}$ such that the comparison axiom is satisfied, i.e., for all $x, y \in \triangle$ and all comparison points $\bar{x}, \bar{y} \in \triangle$, we have

$$
d(x, y) \leq\|\bar{x}-\bar{y}\| .
$$

We will need some known facts about $\ell_{p}$, for $p \geq 2$. The following technical lemma was discovered initially by Lim [23] (see also [32]):

Lemma 1.2. [23] Let $\gamma, \beta \in[0,1]$ be such that $\gamma+\beta=1$. For any $x, y \in \ell_{p}$, we have

$$
\|\gamma x+\beta y\|^{p}+g(\gamma)\|x-y\|^{p} \leq \gamma\|x\|^{p}+\beta\|y\|^{p},
$$


where

$$
g(\gamma)=\gamma \beta \frac{1+[x(\gamma \wedge \beta)]^{p-1}}{[1+x(\gamma \wedge \beta)]^{p-1}},
$$

where $x(\gamma)$, for $\gamma \in[0,1 / 2]$ is the unique solution to

$$
(1-\gamma) x^{p-1}-\gamma-((1-\gamma) x-\gamma)^{p-1}=0, \quad x \in\left[\frac{\gamma}{1-\gamma}, 1\right] .
$$

In particular, we have

$$
g(\gamma) \geq \gamma \beta \frac{1}{2^{p-1}}
$$

which implies

$$
\|\gamma x+\beta y\|^{p}+\frac{\gamma \beta}{2^{p-1}}\|x-y\|^{p} \leq \gamma\|x\|^{p}+\beta\|y\|^{p},
$$

Let $x_{i} \in X$ and $t_{i} \in[0,1]$ for $i=1,2, \ldots, n$ such that $\sum_{i=1}^{n} t_{i}=1$. Following the definition of unique point $(1-\gamma) x \oplus \gamma y$ on a geodesic segment $[x, y]$, we build the following notations: $\bigoplus_{i=1}^{2} t_{i} x_{i}=\frac{t_{1}}{t_{1}+t_{2}} x_{1} \oplus \frac{t_{2}}{t_{1}+t_{2}} x_{2}$. By induction, we can write

$$
\bigoplus_{i=1}^{n} t_{i} x_{i}=\left(1-t_{n}\right)\left(\frac{t_{1}}{1-t_{n}} x_{1} \oplus \frac{t_{2}}{1-t_{n}} x_{2} \oplus \cdots \oplus \frac{t_{n-1}}{1-t_{n}} x_{n-1}\right) \oplus t_{n} x_{n} .
$$

Using Lemma 1.2, we get the following:

Lemma 1.3. Let $X$ be a $\operatorname{CAT}_{\mathrm{p}}(0)$ space, with $p \geq 2$, with $x, x_{i} \in X$ and $t_{i} \in[0,1]$ for $i=$ $1,2, \ldots, n(n \geq 2)$ such that $\sum_{i=1}^{n} t_{i}=1$. Then

(i) $d\left(\bigoplus_{i=1}^{n} t_{i} x_{i}, x\right) \leq \sum_{i=1}^{n} t_{i} d\left(x_{i}, x\right)$;

(ii) $d^{p}\left(\bigoplus_{i=1}^{n} t_{i} x_{i}, x\right) \leq \sum_{i=1}^{n} t_{i} d^{p}\left(x_{i}, x\right)-\frac{1}{2^{p-1}} t_{i} t_{j} d^{p}\left(x_{i}, x_{j}\right)$ for $i, j \in\{1,2, \cdots, n\}$.

Proof. (i) is evident. For (ii) we are going to prove by induction on $n$. For $n=2$, let $x_{1}, x_{2}, x_{3} \in X, x=x_{3}$. Consider the comparison triangle $\Delta\left(\bar{x}_{1}, \bar{x}_{2}, \bar{x}_{3}\right)$ in $\ell_{p}$ such that $d\left(x_{i}, x_{j}\right)=\left\|\bar{x}_{i}-\bar{x}_{j}\right\|_{p}$, for $i, j \in\{1,2,3\}$. We have

$$
\begin{aligned}
d^{p}\left((1-t) x_{1} \oplus t x_{2}, x_{3}\right) & \leq\left\|(1-t) \bar{x}_{1}+t \bar{x}_{2}-\bar{x}_{3}\right\|^{p} \\
& =\left\|(1-t)\left(\bar{x}_{1}-\bar{x}_{3}\right)+t\left(\bar{x}_{2}-\bar{x}_{3}\right)\right\|^{p} \\
& \left.\leq(1-t)\left\|\bar{x}_{1}-\bar{x}_{3}\right\|^{p}+t \| \bar{x}_{2}-\bar{x}_{3}\right)\left\|^{p}-\frac{t(1-t)}{2^{p-1}}\right\| \bar{x}_{1}-\bar{x}_{2} \|^{p} \\
& =(1-t) d^{p}\left(x_{1}, x_{3}\right)+t d^{p}\left(x_{2}, x_{3}\right)-\frac{t(1-t)}{2^{p-1}} d^{p}\left(x_{1}, x_{2}\right) .
\end{aligned}
$$

Inductively, suppose that result holds for $n=k-1$, i.e.,

$$
d^{p}\left(\bigoplus_{i=1}^{k-1} t_{i} x_{i}, x\right) \leq \sum_{i=1}^{k-1} t_{i} d^{p}\left(x_{i}, x\right)-\frac{1}{2^{p-1}} t_{i} t_{j} d^{p}\left(x_{i}, x_{j}\right),
$$

for $i, j \in\{1,2, \cdots, n-1\}$, where $\sum_{i=1}^{k-1} t_{i}=1$. 
Now choose $\left\{t_{1}, t_{2}, \ldots, t_{k}\right\} \subset[0,1]$ with $t_{k} \neq 0$ and such that $\sum_{i=1}^{k} t_{i}=1$. Select $i, j \in$ $\{1, \ldots, k\}$ s.t $i \neq k \neq j$ (we can change position of $t_{i}$ ). By (1.2),

$$
\begin{aligned}
d^{p}\left(\bigoplus_{i=1}^{k} t_{i} x_{i}, x\right) & =d^{p}\left(\left(1-t_{k}\right)\left(\bigoplus_{i=1}^{k-1} \frac{t_{i}}{1-t_{k}} x_{i}\right) \oplus t_{k} x_{k}, x\right) \\
& \leq\left(1-t_{k}\right) d^{p}\left(\left(\bigoplus_{i=1}^{k-1} \frac{t_{i}}{1-t_{k}} x_{i}\right), x\right)+t_{k} d^{p}\left(x_{k}, x\right) \\
& \leq\left(1-t_{k}\right) \sum_{i=1}^{k-1} \frac{t_{i}}{1-t_{k}} d^{p}\left(x_{i}, x\right)+t_{k} d^{p}\left(x_{k}, x\right)-\frac{1-t_{k}}{2^{p-1}} t_{i} t_{j} d^{p}\left(x_{i}, x_{j}\right) \\
& \leq \sum_{i=1}^{k} \frac{t_{i}}{1-t_{k}} d^{p}\left(x_{i}, x\right)-\frac{t_{i} t_{j}}{2^{p-1}} d^{p}\left(x_{i}, x_{j}\right)
\end{aligned}
$$

$i, j \in\{1,2, \cdots, n-1\}$.

From Lemma 1.3, if $p \geq 2$, inequality (1.1) is satisfied for $c=2^{p-2}$. Thus any $\operatorname{CAT}_{\mathrm{p}}(0)$ space is $p$-uniformly convex.

In the proof of the main results we shall use the next Lemma.

Lemma 1.4 ([31]). Suppose that $\left\{a_{n}\right\}$ and $\left\{b_{n}\right\}$ are sequence of nonnegative real numbers such that $a_{n+1} \leq a_{n}+b_{n}$ for all $n \geq N_{0}$, for a fixed $N_{0}$. If $\sum_{n=1}^{\infty} b_{n}<\infty$, then $\lim _{n \rightarrow \infty} a_{n}$ exists.

\section{MAIN RESUlTS}

The main tool for approximation of fixed points of nonexpansive mappings remains iterative technique. Several authors have extensively studied the iterative techniques for nonexpansive in Banach spaces, including the Mann type (one step), the Ishikawa type (two steps), and the three-step iteration processes (see [5]). Agarwal et al. [1] introduced the following iterative process in Banach spaces:

$$
\left\{\begin{array}{l}
x_{n+1}=\left(1-\gamma_{n}\right) T x_{n}+\gamma_{n} T y_{n} \\
y_{n}=\left(1-\beta_{n}\right) x_{n}+\beta_{n} T x_{n}
\end{array} \quad n=1,2,3, \ldots,\right.
$$

In CAT(0) spaces Dhompongsa and Panyanak [11] studied the convergence of the Picard, Mann and Ishikawa iterates.

On the other hand, Liu [24] introduced Ishikawa and Mann iteration methods with errors (see also [34]). We note that, in applications, there are perturbations always occurring in the iterative processes because the manipulations are inaccurate. It is no doubt that researching the convergent problems of iterative methods with perturbation members is a significant job. Recently, Calderon et al. [7] presented a pertubed iteration in CAT $(0)$ spaces.

This leads us, in this paper, to introduce and study a new class of two-step iterative scheme with perturbations for solving the fixed point problem for nonexpansive mappings. This iterative scheme can be viewed as an extension for type iterative schemes of Agarwal et al. [1] adapted it into $\operatorname{CAT}_{\mathrm{p}}(0)$. Here we introduce a perturbation in the model. Consider $K$ to be a nonempty closed convex subset of a complete $\operatorname{CAT}_{\mathrm{p}}(0)$ space $X$ and $T: K \rightarrow K$ be a nonexpansive mapping. Fix $x_{0} \in K$. Consider $\left\{x_{n}\right\}$ defined by the iteration

$$
\left\{\begin{array}{l}
z_{n}=T_{3} x_{n}, \\
y_{n}=\left(1-\beta_{n}-\beta_{n}^{\prime}\right) x_{n} \oplus \beta_{n} T_{2} z_{n} \oplus \beta_{n}^{\prime} \varepsilon_{n}^{\prime}, \\
x_{n+1}=\left(1-\gamma_{n}-\gamma_{n}^{\prime}\right) T_{2} x_{n} \oplus \gamma_{n} T_{1} y_{n} \oplus \gamma_{n}^{\prime} \varepsilon_{n},
\end{array}\right.
$$

for $n \geq 1$, where $\left\{\epsilon_{n}\right\}$ and $\left\{\epsilon_{n}^{\prime}\right\}$ are bounded sequences in $K$ and $\left\{\gamma_{n}\right\}_{n=1}^{\infty},\left\{\beta_{n}\right\}_{n=1}^{\infty}$, $\left\{\gamma_{n}^{\prime}\right\}_{n=1}^{\infty}$ and $\left\{\beta_{n}^{\prime}\right\}_{n=1}^{\infty}$ are appropriate real sequences in $[0,1]$. 
Lemma 2.5. Let $(X, d)$ be a complete $\operatorname{CAT}_{\mathrm{p}}(0)$ space, with $p \geq 2$. Let $K$ be a nonempty closed convex bounded subset of $X$. Let $T_{1}, T_{2}, T_{3}: K \rightarrow K$ be three nonexpansive mappings such that $\Omega=F\left(T_{1}\right) \cap F\left(T_{2}\right) \cap F\left(T_{3}\right) \neq \emptyset$. Let $\left\{x_{n}\right\}$ be the sequence defined by (SM) such that

C1) $\sum_{n=1}^{\infty} \gamma_{n}^{\prime}<\infty$ and $\sum_{n=1}^{\infty} \beta_{n}^{\prime}<\infty$.

C2) $0<\gamma \leq \beta_{n} \leq \beta_{n}+\beta_{n}^{\prime} \leq \beta<1$.

C3) $\gamma \leq \gamma_{n}+\gamma_{n}^{\prime}$.

Then the following hold:

(i) $\lim _{n \rightarrow \infty} d\left(x_{n}, x^{*}\right)$ exists, for any $x^{*}$ of $\Omega$.

(ii) $\lim _{n \rightarrow \infty} d\left(x_{n}, T_{i} x_{n}\right)=0$, for $i=1,2,3$, i.e., $\left\{x_{n}\right\}$ is an approximate fixed point sequence of $T_{i}$, for $i=1,2,3$.

Proof. Let $x^{*} \in \Omega$. Using Lemma 1.3, we get

$$
\begin{aligned}
& d\left(z_{n}, x^{*}\right)=d\left(T_{3} x_{n}, x^{*}\right) \leq d\left(x_{n}, x^{*}\right) \\
d\left(y_{n}, x^{*}\right)= & d\left(\left(1-\beta_{n}-\beta_{n}^{\prime}\right) x_{n} \oplus \beta_{n} T_{2} z_{n} \oplus \beta_{n}^{\prime} \varepsilon_{n}^{\prime}, x^{*}\right) \\
\leq & \left(1-\beta_{n}-\beta_{n}^{\prime}\right) d\left(x_{n}, x^{*}\right)+\beta_{n} d\left(T_{2} z_{n}, x^{*}\right)+\beta_{n}^{\prime} d\left(\varepsilon_{n}^{\prime}, x^{*}\right) \\
\leq & \left(1-\beta_{n}^{\prime}\right) d\left(x_{n}, x^{*}\right)+\beta_{n}^{\prime} d\left(\varepsilon_{n}^{\prime}, x^{*}\right) \\
\leq & d\left(x_{n}, x^{*}\right)+\beta_{n}^{\prime} d\left(\varepsilon_{n}^{\prime}, x^{*}\right)
\end{aligned}
$$

and

$$
\begin{aligned}
d\left(x_{n+1}, x^{*}\right) & =d\left(\left(1-\gamma_{n}-\gamma_{n}^{\prime}\right) T_{2} x_{n} \oplus \gamma_{n} T_{1} y_{n} \oplus \gamma_{n}^{\prime} \varepsilon_{n}, x^{*}\right) \\
& \leq\left(1-\gamma_{n}-\gamma_{n}^{\prime}\right) d\left(T_{2} x_{n}, x^{*}\right)+\gamma_{n} d\left(T_{1} y_{n}, x^{*}\right)+\gamma_{n}^{\prime} d\left(\varepsilon_{n}, x^{*}\right) \\
& \leq\left(1-\gamma_{n}-\gamma_{n}^{\prime}\right) d\left(x_{n}, x^{*}\right)+\gamma_{n} d\left(y_{n}, x^{*}\right)+\gamma_{n}^{\prime} d\left(\varepsilon_{n}, x^{*}\right) \\
& \leq\left(1-\gamma_{n}^{\prime}\right) d\left(x_{n}, x^{*}\right)+\gamma_{n} \beta_{n}^{\prime} d\left(\varepsilon_{n}^{\prime}, x^{*}\right)+\gamma_{n}^{\prime} d\left(\varepsilon_{n}, x^{*}\right) \\
& \leq d\left(x_{n}, x^{*}\right)+\gamma_{n} \beta_{n}^{\prime} d\left(\varepsilon_{n}^{\prime}, x^{*}\right)+\gamma_{n}^{\prime} d\left(\varepsilon_{n}, x^{*}\right),
\end{aligned}
$$

for any $n \geq 1$. Lemma 1.4 implies the conclusion of $(i)$.

Let us prove $(i i)$. Set $\lim _{n \rightarrow \infty} d\left(x_{n}, x^{*}\right)=m$. It is clear that if $m=0$, then $(i i)$ holds.

Otherwise we assume $m>0$. Let us show that $\lim _{n \rightarrow \infty} d\left(y_{n}, x^{*}\right)=m$. We have

$$
d\left(x_{n+1}, x^{*}\right) \leq\left(1-\gamma_{n}-\gamma_{n}^{\prime}\right) d\left(x_{n}, x^{*}\right)+\gamma_{n} d\left(y_{n}, x^{*}\right)+\gamma_{n}^{\prime} d\left(\varepsilon_{n}, x^{*}\right),
$$

which implies

$$
\left(\gamma_{n}+\gamma_{n}^{\prime}\right) d\left(x_{n}, x^{*}\right) \leq \gamma_{n} d\left(y_{n}, x^{*}\right)+d\left(x_{n}, x^{*}\right)-d\left(x_{n+1}, x^{*}\right)+\gamma_{n}^{\prime} d\left(\varepsilon_{n}, x^{*}\right)
$$

or

$$
\begin{aligned}
d\left(x_{n}, x^{*}\right) & \leq \frac{\gamma_{n}}{\gamma_{n}+\gamma_{n}^{\prime}} d\left(y_{n}, x^{*}\right)+\frac{1}{\gamma_{n}+\gamma_{n}^{\prime}}\left[d\left(x_{n}, x^{*}\right)-d\left(x_{n+1}, x^{*}\right)\right]+\frac{\gamma_{n}^{\prime}}{\gamma_{n}+\gamma_{n}^{\prime}} d\left(\varepsilon_{n}, x^{*}\right) \\
& \leq d\left(y_{n}, x^{*}\right)+\frac{1}{\gamma}\left[d\left(x_{n}, x^{*}\right)-d\left(x_{n+1}, x^{*}\right)\right]+\frac{\gamma_{n}^{\prime}}{\gamma} d\left(\varepsilon_{n}, x^{*}\right),
\end{aligned}
$$

for any $n \geq 1$. Therefore we have

$$
\begin{aligned}
m & =\liminf _{n \rightarrow \infty} d\left(x_{n} \cdot x^{*}\right) \\
& \leq \liminf _{n \rightarrow \infty} d\left(y_{n}, x^{*}\right)+\liminf _{n \rightarrow \infty} \frac{1}{\gamma}\left[d\left(x_{n}, x^{*}\right)-d\left(x_{n+1}, x^{*}\right)\right]+\liminf _{n \rightarrow \infty} \frac{\gamma_{n}^{\prime}}{\gamma} d\left(\varepsilon_{n}, x^{*}\right) \\
& =\liminf _{n \rightarrow \infty} d\left(y_{n}, x^{*}\right) .
\end{aligned}
$$


Similarly, we prove limsup $d\left(y_{n}, x^{*}\right) \leq m$, which implies

$$
\lim _{n \rightarrow \infty} d\left(y_{n}, x^{*}\right)=m .
$$

Similarly, we have

$$
\begin{aligned}
d\left(y_{n}, x^{*}\right) & =d\left(\left(1-\beta_{n}-\beta_{n}^{\prime}\right) x_{n} \oplus \beta_{n} T_{2} z_{n} \oplus \beta_{n}^{\prime} \varepsilon_{n}^{\prime}, x^{*}\right) \\
& \leq\left(1-\beta_{n}-\beta_{n}^{\prime}\right) d\left(x_{n}, x^{*}\right)+\beta_{n} d\left(T_{2} z_{n}, x^{*}\right)+\beta_{n}^{\prime} d\left(\varepsilon_{n}^{\prime}, x^{*}\right) \\
& \leq\left(1-\beta_{n}-\beta_{n}^{\prime}\right) d\left(x_{n}, x^{*}\right)+\beta_{n} d\left(z_{n}, x^{*}\right)+\beta_{n}^{\prime} d\left(\varepsilon_{n}^{\prime}, x^{*}\right),
\end{aligned}
$$

which implies that

$$
\left(\beta_{n}+\beta_{n}^{\prime}\right) d\left(x_{n}, x^{*}\right) \leq\left(d\left(x_{n}, x^{*}\right)-d\left(y_{n}, x^{*}\right)\right)+\beta_{n} d\left(z_{n}, x^{*}\right)+\beta_{n}^{\prime} d\left(\varepsilon_{n}^{\prime}, x^{*}\right),
$$

or

$$
\begin{aligned}
d\left(x_{n}, x^{*}\right) & \leq \frac{1}{\beta_{n}+\beta_{n}^{\prime}}\left(d\left(x_{n}, x^{*}\right)-d\left(y_{n}, x^{*}\right)\right)+\frac{\beta_{n}}{\beta_{n}+\beta_{n}^{\prime}} d\left(z_{n}, x^{*}\right)+\frac{\beta_{n}^{\prime}}{\beta_{n}+\beta_{n}^{\prime}} d\left(\varepsilon_{n}^{\prime}, x^{*}\right) \\
& \leq \frac{1}{\gamma}\left(d\left(x_{n}, x^{*}\right)-d\left(y_{n}, x^{*}\right)\right)+d\left(z_{n}, x^{*}\right)+\frac{\beta_{n}^{\prime}}{\gamma} d\left(\varepsilon_{n}^{\prime}, x^{*}\right),
\end{aligned}
$$

and $m=\liminf _{n \rightarrow \infty} d\left(x_{n}, x^{*}\right) \leq \liminf _{n \rightarrow \infty} d\left(z_{n}, x^{*}\right)$. Similary $\lim \sup _{n \rightarrow \infty} d\left(z_{n}, x^{*}\right) \leq$ $\limsup _{n \rightarrow \infty} d\left(x_{n}, x^{*}\right)=m$. Thus $\lim _{n \rightarrow \infty} d\left(z_{n}, x^{*}\right)=m$. This shows that

$$
\lim _{n \rightarrow \infty} d\left(x_{n}, z_{n}\right)=0 .
$$

On other hand, using Lemma 1.3, we get

$$
\begin{aligned}
d^{p}\left(y_{n}, x^{*}\right) & =d^{p}\left(\left(1-\beta_{n}-\beta_{n}^{\prime}\right) x_{n} \oplus \beta_{n} T_{2} z_{n} \oplus \beta_{n}^{\prime} \varepsilon_{n}^{\prime}, x^{*}\right) \\
& \leq\left(1-\beta_{n}-\beta_{n}^{\prime}\right) d^{p}\left(x_{n}, x^{*}\right)+\beta_{n} d^{p}\left(T_{2} z_{n}, x^{*}\right)+\beta_{n}^{\prime} d^{p}\left(\varepsilon_{n}^{\prime}, x^{*}\right) \\
& -\frac{\beta_{n}\left(1-\beta_{n}-\beta_{n}^{\prime}\right)}{2^{p-1}} d^{p}\left(x_{n}, T_{2} z_{n}\right) \\
& \leq d^{p}\left(x_{n}, x^{*}\right)+\beta_{n}^{\prime} d^{p}\left(\varepsilon_{n}^{\prime}, x^{*}\right)-\frac{\beta_{n}\left(1-\beta_{n}-\beta_{n}^{\prime}\right)}{2^{p-1}} d^{p}\left(x_{n}, T_{2} z_{n}\right)
\end{aligned}
$$

Since $\left\{\beta_{n}, \beta_{n}+\beta_{n}^{\prime}\right\} \subset[\gamma, \beta]$, we get

$$
\begin{aligned}
\frac{\gamma(1-\beta)}{2^{p-1}} d^{p}\left(x_{n}, T_{2} z_{n}\right) & \leq \frac{\beta_{n}\left(1-\beta_{n}-\beta_{n}^{\prime}\right)}{2^{p-1}} d^{p}\left(x_{n}, T_{2} z_{n}\right) \\
& \leq \beta_{n}^{\prime} d^{p}\left(\varepsilon_{n}^{\prime}, x^{*}\right)+d^{p}\left(x_{n}, x^{*}\right)-d^{p}\left(y_{n}, x^{*}\right),
\end{aligned}
$$

which implies

$$
d^{p}\left(x_{n}, T_{2} z_{n}\right) \leq \frac{2^{p-1}}{\gamma(1-\beta)}\left[\beta_{n}^{\prime} d^{p}\left(\varepsilon_{n}^{\prime}, x^{*}\right)+d^{p}\left(x_{n}, x^{*}\right)-d^{p}\left(y_{n}, x^{*}\right)\right],
$$

for any $n \geq 1$. Clearly, our previous results will force

$$
\lim _{n \rightarrow \infty} d\left(x_{n}, T_{2} z_{n}\right)=0 .
$$

It follows from (2.4 and (2.3) that

$$
d\left(x_{n}, T_{2} x_{n}\right) \leq d\left(x_{n}, T_{2} z_{n}\right)+d\left(T_{2} z_{n}, T_{2} x_{n}\right) \leq d\left(x_{n}, T_{2} z_{n}\right)+d\left(z_{n}, x_{n}\right) \rightarrow 0 .
$$

Using similar arguments, $\lim _{n \rightarrow \infty} d\left(x_{n}, T_{1} x_{n}\right)=0$, which completes the proof of Lemma 2.5.

Remark 2.1. (1) From [20], we know that if $T$ is nonexpansive, then $T$ has a nonempty fixed point set $F(T)$. If $T_{1}=T_{2}=T_{3}=T$ in Lemma 2.5, then we obtain that $\Omega=F(T)$. 
(2) We can prove Lemma 2.5 so far in the context of quasi-nonexpansive mappings with suitable changes. Moreover, it is ease prove Lemma 2.5 combining nonexpansive mappings with quasi-nonexpansive mappings.

Next, we give the main result of our work.

Theorem 2.1. Let $X, K, T_{1}, T_{2}, T_{3},\left\{x_{n}\right\}$ satisfy the hypothesis of Lemma 2.5. Then the sequence $\left\{x_{n}\right\}$ defined by $(S M) \Delta$-converges to a point of $\Omega$.

Proof. By Lemma 2.5, we know that $\lim _{n \rightarrow \infty} d\left(x_{n}, T_{i} x_{n}\right)=0$, for $i=1,2,3$ and $\lim _{n \rightarrow \infty} d\left(x_{n}, x^{*}\right)$ exists, for any $x^{*} \in \Omega$. Hence $\left\{x_{n}\right\}$ is bounded. We first show that $w_{\Delta}\left(x_{n}\right) \subseteq \Omega$, where $w_{\Delta}\left(x_{n}\right)$ is the set of the asymptotic centers of all subsequences of $\left\{x_{n}\right\}$. Let $u \in w_{\Delta}\left(x_{n}\right)$, then there exists a subsequence $\left\{u_{n}\right\}$ of $\left\{x_{n}\right\}$ such that $A\left(\left\{u_{n}\right\}\right)=\{u\}$. By Lemma 1.1, there exists a subsequence $\left\{v_{n}\right\}$ of $\left\{u_{n}\right\}$ such that $\Delta-\lim _{n} v_{n}=v \in K$. By Lemma 1.1, $v \in \Omega$. Now, from Lemma 2.5, $\lim d\left(x_{n}, v\right)$ exists. We now claim that $u=v$. Assume on the contrary, that $u \neq v$. Then, by the uniqueness of asymptotic centers we have

$$
\begin{aligned}
& \limsup _{n \rightarrow \infty} d\left(v_{n}, v\right)<\limsup _{n \rightarrow \infty} d\left(v_{n}, u\right) \leq \limsup _{n \rightarrow \infty} d\left(u_{n}, u\right) \\
& <\limsup _{n \rightarrow \infty} d\left(u_{n}, v\right) \quad=\limsup _{n \rightarrow \infty} d\left(x_{n}, v\right) \\
& =\limsup _{n \rightarrow \infty} d\left(v_{n}, v\right)
\end{aligned}
$$

Which is a contradiction. Thus, $u=v \in \Omega$ and hence $w_{\Delta}\left(x_{n}\right) \subseteq \Omega$ consists of exactly one point. Let $\left\{u_{n}\right\}$ be a subsequence of $\left\{x_{n}\right\}$. By Lemma 1.1, there exists a subsequence $\left\{v_{n}\right\}$ of $\left\{u_{n}\right\}$ such that $\Delta-\lim _{n} v_{n}=v \in K$. Let $A\left(\left\{u_{n}\right\}\right)=\{u\}$ and $A\left(\left\{x_{n}\right\}\right)=\{x\}$. We have already seen that $u=v$ and $v \in \Omega$. Finally, we claim that $x=v$. If not, then the existence of $\lim _{n \rightarrow \infty} d\left(x_{n}, v\right)$ and uniqueness of asymptotic centers imply that

$$
\begin{aligned}
\limsup _{n \rightarrow \infty} d\left(v_{n}, v\right)<\limsup _{n \rightarrow \infty} d\left(v_{n}, x\right) & \leq \limsup _{n \rightarrow \infty} d\left(x_{n}, x\right) \\
<\limsup _{n \rightarrow \infty} d\left(x_{n}, v\right) & =\limsup _{n \rightarrow \infty} d\left(v_{n}, v\right) .
\end{aligned}
$$

Again, it is a contradiction, hence $x=v \in \Omega$. Therefore, $w_{\Delta}\left(x_{n}\right)=\{x\}$.

Theorem 2.2. Let $X, K, T_{1}, T_{2}, T_{3},\left\{x_{n}\right\}$ satisfy the hypothesis of Lemma 2.5. Then the sequence $\left\{x_{n}\right\}$ defined by $(S M)$

i) $\Delta$-converges to a point of $\Omega$.

ii) converges strongly to a fixed point of $T$ if and only if $\liminf _{n \rightarrow \infty} d\left(x_{n}, \Omega\right)=0$, where $d(x, \Omega)=\inf \left\{d\left(x, x^{*}\right): x^{*} \in \Omega\right\}$.

Proof. The necessity is obvius. To prove the converse, suppose that

$$
\liminf _{n \rightarrow \infty} d\left(x_{n}, \Omega\right)=0 .
$$

Thus by hypothesis $\lim _{n \rightarrow \infty} d\left(x_{n}, \Omega\right)=0$. Next, we show that $\left\{x_{n}\right\}$ is Cauchy sequence in $K$. Let $\epsilon>0$ be arbitrarily chosen. Since $\lim _{n \rightarrow \infty} d\left(x_{n}, \Omega\right)=0$, there exists a positive integer $n_{0}$ such that

$$
d\left(x_{n}, \Omega\right)<\frac{\epsilon}{4}, \forall n \geq n_{0} .
$$

In particular, $\inf \left\{d\left(x_{n_{0}}, x^{*}\right): x^{*} \in \Omega\right\}<\frac{\epsilon}{4}$. Thus there must exist $x^{* *} \in \Omega$ such that

$$
d\left(x_{n_{0}}, x^{* *}\right)<\frac{\epsilon}{2} .
$$

Now, for all $m, n \geq n_{0}$, we have 


$$
\begin{aligned}
d\left(x_{n+m}, x_{n}\right) & \leq d\left(x_{n+m}, x^{* *}\right)+d\left(x^{* *}, x_{n}\right) \\
& \leq 2 d\left(x_{n_{0}}, x^{* *}\right) \\
& \leq 2\left(\frac{\epsilon}{2}\right)=\epsilon
\end{aligned}
$$

This proves that $\left\{x_{n}\right\}$ is a Cauchy sequence in $K$. Thus, the completeness of $X$ implies that $\left\{x_{n}\right\}$ must be convergence. Since $\lim _{n \rightarrow \infty} d\left(x_{n}, \Omega\right)=0$, we get $d\left(x^{*}, \Omega\right)=0$, closedness of $\Omega$ gives that $x^{*} \in \Omega$. Thus $\left\{x_{n}\right\}$ converges strongly to a point in $\Omega$. This completes the proof.

\section{Applications}

3.1. Proximal Point Algorithm in $C A T(0)$ spaces. Recall that a function $f: C \rightarrow(-\infty, \infty]$ defined on a convex subset $C$ of a $C A T(0)$ space is convex if, for any geodesic $\gamma:[a, b] \rightarrow$ $C$, the function $f \gamma$ is convex. Some important examples can be found in [6]. We say that a function $\mathrm{f}$ defined on $C$ is lower semi-continuous at a point $x \in C$ if $f(x) \leq \liminf _{n \rightarrow \infty} f\left(x_{n}\right)$, for each sequence $x_{n} \rightarrow x$. A function $\mathrm{f}$ is said to be lower semi-continuous on $C$ if it is lower semi-continuous at any point in $C$.

For any $\lambda>0$, define the Moreau-Yosida resolvent of $f$ in $C A T(0)$ spaces as

$$
J_{\lambda}(x)=\operatorname{argmin}_{y \in X}\left[f(y)+\frac{1}{2 \lambda} d^{2}(y, x)\right],
$$

for all $x \in X$. Originally, this definition is due to Moreau [26]. The mapping $J_{\lambda}$ is welldefined for every $\lambda>0$ (see [14, 25]).

Let $f: X \rightarrow(-\infty, \infty]$ be be a proper convex and lower semi-continuous function. It was shown in [3] that the set $F\left(J_{\lambda}\right)$ of fixed points of the resolvent associated with $f$ coincides with the set $\operatorname{argmin}_{y \in X} f(y)$ of minimizers of $f$.

Lemma 3.6. [14] Let $(X, d)$ be a complete $C A T(0)$ space and $f: X \rightarrow(-\infty, \infty]$ be proper convex and lower semi-continuous. For any $\lambda>0$, the resolvent $J_{\lambda}$ of $f$ is nonexpansive.

Taking $T_{3}=J_{\lambda_{n}}$ in (SM) iteration, we obtain the next result in $C A T(0)$ spaces as a direct consequence of Theorem 2.1.

Theorem 3.3. Let $(X, d)$ be a complete $\mathrm{CAT}(0)$ space. Let $K$ be a nonempty closed convex bounded subset of $X$. Let $f: X \rightarrow(-\infty, \infty]$ be proper convex and lower semi-continuous. Let $T_{1}, T_{2}: K \rightarrow K$ be two nonexpansive mappings such that $\Omega=F\left(T_{1}\right) \cap F\left(T_{2}\right) \cap \operatorname{argmin}_{y \in X} f(y) \neq$ $\emptyset$. Let $\left\{x_{n}\right\}$ be the sequence defined by

(SM0)

$$
\left\{\begin{array}{l}
z_{n}=J_{\lambda_{n}} x_{n}, \\
y_{n}=\left(1-\beta_{n}-\beta_{n}^{\prime}\right) x_{n} \oplus \beta_{n} T_{2} z_{n} \oplus \beta_{n}^{\prime} \varepsilon_{n}^{\prime}, \\
x_{n+1}=\left(1-\gamma_{n}-\gamma_{n}^{\prime}\right) T_{2} x_{n} \oplus \gamma_{n} T_{1} y_{n} \oplus \gamma_{n}^{\prime} \varepsilon_{n},
\end{array}\right.
$$

for $n \geq 1$, where $\left\{\epsilon_{n}\right\}$ and $\left\{\epsilon_{n}^{\prime}\right\}$ are bounded sequences in $K$ and $\left\{\gamma_{n}\right\}_{n=1}^{\infty},\left\{\beta_{n}\right\}_{n=1}^{\infty},\left\{\gamma_{n}^{\prime}\right\}_{n=1}^{\infty}$ and $\left\{\beta_{n}^{\prime}\right\}_{n=1}^{\infty}$ are appropriate real sequences in $[0,1]$ and $\left\{\lambda_{n}\right\}_{n=1}^{\infty}$ is a sequence in $\mathbb{R}$ such that

C1) $\sum_{n=1}^{\infty} \gamma_{n}^{\prime}<\infty$ and $\sum_{n=1}^{\infty} \beta_{n}^{\prime}<\infty$.

C2) $0<\gamma \leq \beta_{n} \leq \beta_{n}+\beta_{n}^{\prime} \leq \beta<1$.

C3) $\gamma \leq \gamma_{n}+\gamma_{n}^{\prime}$.

C4) $\lambda_{n} \geq \lambda>0$, for some $\lambda$.

Then the sequence $\left\{x_{n}\right\}$ defined by (SMO) $\Delta$-converges to a point of $\Omega$. 
Theorem 3.3 extends that of Cholamjiak et al. [10] in $C A T(0)$ spaces. In fact, we present a new perturbed proximal point algorithm for solving the convex minimization problem as well as the fixed point problem of nonexpansive mappings in $\mathrm{CAT}(0)$ spaces.

3.2. Proximal Point Algorithm in $C A T(\kappa)$ spaces. For a real number $\kappa$, a $C A T(\kappa)$ space is defined by a geodesic space $(X, d)$ whose geodesic triangle is sufficiently thinner than the corresponding comparison triangle in a model space with curvature $\kappa$. The concept of such a space has been investigated by lots of researchers. In 2003, Kirk [17, 18] first proved the existence of fixed points for nonexpansive mappings in a $C A T(\kappa)$ space for $\kappa \geq 0$. In other to be short, for more detailed discussion on them, the reader can consult, for instance, $[6,9,29]$ and references therein. Since many results and basic concepts in $C A T(\kappa)$ can be deduced frome those in $C A T(1)$ spaces, we now sufficiently state useful lemmas and definitions on $C A T(1)$ spaces. Throughout this section, we suppose that $X$ is an admisible $C A T(1)$ space, i.e., $d(x, y)<\frac{\pi}{2}$, for all $x, y \in X$.

Let $(X, d)$ be a $C A T(1)$ space. Let $f: X \rightarrow(-\infty, \infty]$ be be a proper convex and lower semi-continuous function. For any $\lambda>0$, define the Kimura-Kohsaka resolvent of $f$ (see $[15,16])$ in $C A T(1)$ spaces as

$$
R_{\lambda}(x)=\operatorname{argmin}_{y \in X}\left[f(y)+\frac{1}{\lambda} \tan d(y, x) \sin d(y, x)\right],
$$

for all $x \in X$. The mapping $R_{\lambda}$ is well defined for all $\lambda>0$ (see $[15,16]$ ) and the set $F\left(R_{\lambda}\right)$ of fixed points of the resolvent associated with $f$ coincides with the set $\operatorname{argmin}_{y \in X} f(y)$ of minimizers of $f$.

Lemma 3.7. [16] Let $(X, d)$ be an admissible complete $C A T(1)$ space and $f: X \rightarrow(-\infty, \infty]$ be proper convex and lower semi-continuous. For any $\lambda>0$, the resolvent $R_{\lambda}$ of $f$ is quasinonexpansive.

Taking $T_{3}=R_{\lambda_{n}}$ in (SM) iteration, we obtain the next result in $C A T(1)$ spaces as a direct consequence of Theorem 2.1 and Remark 2.1.

Theorem 3.4. Let $(X, d)$ be an admissible complete CAT(1) space. Let $K$ be a nonempty closed convex bounded subset of $X$. Let $f: X \rightarrow(-\infty, \infty]$ be proper convex and lower semi-continuous. Let $T_{1}, T_{2}: K \rightarrow K$ be two quasi-nonexpansive mappings such that $\Omega=F\left(T_{1}\right) \cap F\left(T_{2}\right) \cap$ $\operatorname{argmin}_{y \in X} f(y) \neq \emptyset$. Let $\left\{x_{n}\right\}$ be the sequence defined by

$$
\left\{\begin{array}{l}
z_{n}=R_{\lambda_{n}} x_{n}, \\
y_{n}=\left(1-\beta_{n}-\beta_{n}^{\prime}\right) x_{n} \oplus \beta_{n} T_{2} z_{n} \oplus \beta_{n}^{\prime} \varepsilon_{n}^{\prime}, \\
x_{n+1}=\left(1-\gamma_{n}-\gamma_{n}^{\prime}\right) T_{2} x_{n} \oplus \gamma_{n} T_{1} y_{n} \oplus \gamma_{n}^{\prime} \varepsilon_{n},
\end{array}\right.
$$

for $n \geq 1$, where $\left\{\epsilon_{n}\right\}$ and $\left\{\epsilon_{n}^{\prime}\right\}$ are bounded sequences in $K$ and $\left\{\gamma_{n}\right\}_{n=1}^{\infty},\left\{\beta_{n}\right\}_{n=1}^{\infty},\left\{\gamma_{n}^{\prime}\right\}_{n=1}^{\infty}$ and $\left\{\beta_{n}^{\prime}\right\}_{n=1}^{\infty}$ are appropriate real sequences in $[0,1]$ and $\left\{\lambda_{n}\right\}_{n=1}^{\infty}$ is a sequence in $\mathbb{R}$ such that

C1) $\sum_{n=1}^{\infty} \gamma_{n}^{\prime}<\infty$ and $\sum_{n=1}^{\infty} \beta_{n}^{\prime}<\infty$.

C2) $0<\gamma \leq \beta_{n} \leq \beta_{n}+\beta_{n}^{\prime} \leq \beta<1$.

C3) $\gamma \leq \gamma_{n}+\gamma_{n}^{\prime}$.

C4) $\lambda_{n} \geq \lambda>0$, for some $\lambda$.

Then the sequence $\left\{x_{n}\right\}$ defined by (SM1) $\Delta$-converges to a point of $\Omega$.

Theorem 3.4 is also valid for $T_{1}, T_{2}: K \rightarrow K$ be two nonexpansive mappings and, in this sense, Theorem 3.4 extends that of Pakkatanang et al. [29, 30] in $C A T(1)$ spaces for quasi-nonexpansive mappings. In fact, we present a new perturbed proximal point algorithm for solving the convex minimization problem as well as the fixed point problem of 
nonexpansive mappings in CAT(1) spaces. A version for $\kappa>0$ can be deduced following [29].

3.3. Fixed point results for $\alpha$-nonexpansive mappings. Inspired by Goebel and Japón Pineda definiton in Banach space [13], we say that

Definition 3.4. Let $X$ be a $\operatorname{CAT}_{\mathrm{p}}(0)$ space. $T: X \rightarrow X$ is mean nonexpansive (or $\alpha$-nonexpansive) if, for some multi-index $\alpha=\left(\alpha_{1}, \ldots, \alpha_{n}\right)$ with $\alpha_{1}, \alpha_{n}>0, \alpha_{j} \geq 0$ for all $j$, and $\alpha_{1}+\cdots+$ $\alpha_{n}=1$, we have

$$
\bigoplus_{\mathbf{j}=\mathbf{1}}^{\mathbf{n}} \alpha_{j} d\left(T^{j} x, T^{j} y\right) \leq d(x, y)
$$

for all $x, y \in X$.

Since the first coefficient $\alpha_{1}>0, T$ satisfies the Lipschitz condition

$$
d(T x, T y) \leq \frac{1}{\alpha_{1}} d(x, y) .
$$

Trivially, $T_{\alpha}: X \rightarrow X$ defined by $T_{\alpha}(x)=\oplus_{i=1}^{n} \alpha_{i} T^{i} x$ is a nonexpansive mapping.

In this section, we shall prove some fixed point theorems for $\alpha$-nonexpansive mapping in a $\operatorname{CAT}_{\mathrm{p}}(0)$ spaces $(\mathrm{CAT}(0)$ spaces).

Theorem 3.5. Let $K$ be a closed convex subset of a $\mathrm{CAT}_{\mathrm{p}}(0)$ space. Let $T: K \rightarrow K$ an $\alpha$ nonexpansive mapping with $\alpha=\left(\alpha_{1}, \ldots, \alpha_{n}\right)$ such that $\alpha_{1}>2^{\frac{1}{1-n}}$. Then the set of fixed points of $T, F(T)$, coincides with the set of fixed point of $T_{\alpha}, F\left(T_{\alpha}\right)$.

Proof. Proof is similar to [21, Theorem 3.1] Trivially, $F(T) \subset F\left(T_{\alpha}\right)$. For contrary, we check that

$$
\begin{aligned}
d\left(T^{j} x, T x\right) & \leq \frac{1}{\alpha_{1}} d\left(T^{j-1} x, x\right) \leq \frac{1}{\alpha_{1}}\left(d\left(T^{j-1} x, T x\right)+d(T x, x)\right) \\
& \leq \frac{1}{\alpha_{1}^{2}} d\left(T^{j-2} x, x\right)+\frac{1}{\alpha_{1}} d(T x, x) \\
& \vdots \\
& \leq\left(\frac{1}{\alpha_{1}^{j-1}}+\ldots+\frac{1}{\alpha_{1}^{2}}+\frac{1}{\alpha_{1}}\right) d(T x, x)
\end{aligned}
$$

for $j=2, \ldots, n$.

If $x \in F\left(T_{\alpha}\right)$, then, by Lemma 1.3 and (3.5),

$$
\begin{aligned}
d(x, T x) & =d\left(T_{\alpha}(x), T x\right) \leq \sum_{j=1}^{n} \alpha_{j} d\left(T^{j} x, T x\right) \\
& \leq \sum_{j=2}^{n} \alpha_{j}\left(\frac{1}{\alpha_{1}^{j-1}}+\ldots+\frac{1}{\alpha_{1}^{2}}+\frac{1}{\alpha_{1}}\right) d(T x, x) \\
& =\left(\sum_{j=1}^{n-1} \frac{1-\alpha_{1}-\alpha_{2}-\ldots-\alpha_{j}}{\alpha_{1}^{j}}\right) d(T x, x) \\
& \leq \frac{1-\alpha_{1}^{n-1}}{\alpha_{1}^{n-1}} d(T x, x)
\end{aligned}
$$

Since $\alpha>2^{\frac{1}{1-n}}, \frac{1-\alpha_{1}^{n-1}}{\alpha_{1}^{n-1}}<1$ and $T x=x$. 
Lemma 3.8. In same conditions of Theorem 3.5. Let $\left\{x_{m}\right\}$ be a bounded sequence in $K$. Then $d\left(x_{m}, T x_{m}\right) \rightarrow 0$ if and only if $d\left(x_{m}, T_{\alpha}\left(x_{m}\right)\right) \rightarrow 0$ as $m \rightarrow \infty$.

Proof. Suppose $d\left(x_{m}, T x_{m}\right) \rightarrow 0$ as $m \rightarrow \infty$. By (3.5),

$$
\begin{aligned}
d\left(T^{j} x_{m}, x_{m}\right) & \leq d\left(T^{j} x_{m}, T x_{m}\right)+d\left(T x_{m}, x_{m}\right) \\
& \leq\left(\frac{1}{\alpha_{1}^{j-1}}+\ldots+\frac{1}{\alpha_{1}^{2}}+\frac{1}{\alpha_{1}}+1\right) d\left(T x_{m}, x_{m}\right) \rightarrow 0,
\end{aligned}
$$

as $m \rightarrow \infty$, for $j=2, \ldots, n$. For $j=1$ is trivial. Now,

$$
d\left(T_{\alpha}\left(x_{m}\right), x_{m}\right) \leq \sum_{j=1}^{n} \alpha_{j} d\left(T^{j} x_{m}, x_{m}\right) \rightarrow 0,
$$

as $m \rightarrow \infty$.

Conversely, Suppose $d\left(x_{m}, T_{\alpha}\left(x_{m}\right)\right) \rightarrow 0$ as $m \rightarrow \infty$. Using a similar procedure to (3.6),

$$
\begin{aligned}
d\left(x_{m}, T x_{m}\right) & \leq d\left(x_{m}, T_{\alpha}\left(x_{m}\right)\right)+d\left(T x_{m}, T_{\alpha}\left(x_{m}\right)\right) \\
& \leq d\left(x_{m}, T_{\alpha}\left(x_{m}\right)\right)+\frac{1-\alpha_{1}^{n-1}}{\alpha_{1}^{n-1}} d\left(T x_{m}, x_{m}\right) \rightarrow 0,
\end{aligned}
$$

i.e., $d\left(T x_{m}, x_{m}\right) \leq\left(2-\frac{1}{\alpha_{1}^{n-1}}\right) d\left(x_{m}, T_{\alpha}\left(x_{m}\right)\right) \rightarrow 0$, as $m \rightarrow \infty$.

Corollary 3.1. Let $(X, d)$ be a complete $\mathrm{CAT}_{\mathrm{p}}(0)$ space, with $p \geq 2$. Let $K$ be a nonempty closed convex bounded subset of $X$. Let $T_{1}, T_{2}, T_{3}: K \rightarrow K$ be three $\alpha$-nonexpansive mappings with $\alpha=\left(\alpha_{1}, \ldots, \alpha_{n}\right)$ such that $\alpha_{1}>2^{\frac{1}{1-n}}$ and such that $\Omega=F\left(T_{1}\right) \cap F\left(T_{2}\right) \cap F\left(T_{3}\right) \neq \emptyset$. Let $\left\{x_{n}\right\}$ be the sequence defined by (SM) such that

C1) $\sum_{n=1}^{\infty} \gamma_{n}^{\prime}<\infty$ and $\sum_{n=1}^{\infty} \beta_{n}^{\prime}<\infty$.

C2) $0<\gamma \leq \beta_{n} \leq \beta_{n}+\beta_{n}^{\prime} \leq \beta<1$.

C3) $\gamma \leq \gamma_{n}+\gamma_{n}^{\prime}$.

Then the following hold:

(i) $\lim _{n \rightarrow \infty} d\left(x_{n}, x^{*}\right)$ exists, for any fixed point $x^{*}$ of $T$.

(ii) $\lim _{n \rightarrow \infty} d\left(x_{n}, T_{i} x_{n}\right)=0$, i.e., $\left\{x_{n}\right\}$ is an approximate fixed point sequence of $T_{i}$, for $i=$ $1,2,3$.

Corollary 3.2. Let $X, K, T_{1}, T_{2}, T_{3},\left\{x_{n}\right\}$ satisfy the hypothesis of Corollary 3.1. Then the sequence $\left\{x_{n}\right\}$ defined by (SM) $\Delta$-converges to a point of $\Omega$.

\section{NUMERICAL EXAMPLES.}

In this section, we provide the numerical examples to illustrate its performance and to compare propose iteration with existing methods.

Example 4.1. Consider $X=\mathbb{R}^{2}$ equipped with the Euclidean norm. Let $x=\left(x_{1}, x_{2}\right) \in \mathbb{R}^{2}$, then the squared distance of $x$ from the origin is

$$
\|x\|^{2}=x_{1}^{2}+x_{2}^{2} .
$$

Consider $K$ as the closed unit disk:

$$
K=\left\{\left(x_{1}, x_{2}\right) \in \mathbb{R}^{2}: x_{1}^{2}+x_{2}^{2} \leq 1\right\}
$$


FIGURE 1. Convergence behavior for Ex. 1.

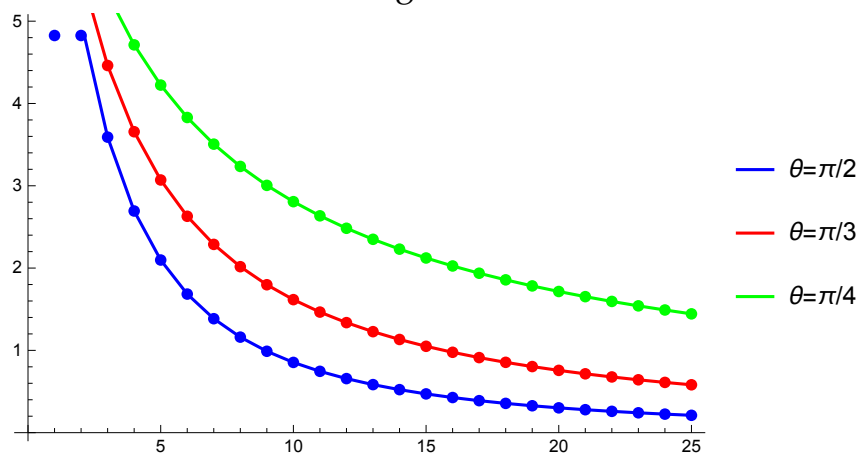

TABLE 1. Error convergence behavior for Ex. 1

\begin{tabular}{l|lll}
\hline Error & $\theta=\pi / 2$ & $\theta=\pi / 3$ & $\theta=\pi / 4$ \\
\hline$\left\|x_{(1)}^{2}\right\|$ & 7.07107 & 7.07107 & 7.07107 \\
$\left\|x_{(3)}^{2}\right\|$ & 3.59117 & 4.46127 & 5.33126 \\
$\left\|x_{(5)}^{2}\right\|$ & 2.09783 & 3.07041 & 4.2235 \\
$\left\|x_{(7)}^{2}\right\|$ & 1.38474 & 2.28779 & 3.50592 \\
$\left\|x_{(9)}^{2}\right\|$ & 0.988957 & 1.79742 & 3.00499 \\
$\left\|x_{(11)}^{2}\right\|$ & 0.745293 & 1.4653 & 2.63483 \\
$\left\|x_{(13)}^{2}\right\|$ & 0.583899 & 1.22732 & 2.34957 \\
$\left\|x_{(15)}^{2}\right\|$ & 0.471072 & 1.04946 & 2.12257 \\
$\left\|x_{(17)}^{2}\right\|$ & 0.388866 & 0.912109 & 1.93738 \\
$\left\|x_{(19)}^{2}\right\|$ & 0.326979 & 0.80324 & 1.78322 \\
$\left\|x_{(21)}^{2}\right\|$ & 0.279137 & 0.715097 & 1.65276 \\
$\left\|x_{(23)}^{2}\right\|$ & 0.241334 & 0.642464 & 1.54085 \\
$\left\|x_{(25)}^{2}\right\|$ & 0.210907 & 0.581713 & 1.44372
\end{tabular}

which is bounded, closed, and convex in $X$. We define mapping $\operatorname{Rot}_{\theta}: K \rightarrow K$ by:

$$
\operatorname{Rot}_{\theta}\left(x_{1}, x_{2}\right)=\left[\begin{array}{cc}
\cos (\theta) & \sin (\theta) \\
-\sin (\theta) & \cos (\theta)
\end{array}\right]\left[\begin{array}{l}
x_{1} \\
x_{2}
\end{array}\right] .
$$

For $\theta=\frac{\pi}{4}$ and $T_{1}=T_{2}=T_{3}=\operatorname{Rot}_{\theta}$, our algorithm is the following:

$$
\left\{\begin{array}{l}
z_{(n)}=\frac{1}{\sqrt{2}}\left(\begin{array}{cc}
1 & 1 \\
-1 & 1
\end{array}\right) x_{(n)} \\
y_{(n)}=\left(\begin{array}{cc}
1-\beta_{n} & \beta_{n} \\
-\beta_{n} & 1-\beta_{n}
\end{array}\right) x_{(n)} \\
x_{(n+1)}=\frac{1}{\sqrt{2}}\left(\begin{array}{cc}
1-2 \gamma_{n} \beta_{n} & 1 \\
-1 & 1-2 \gamma_{n} \beta_{n}
\end{array}\right) x_{(n)}
\end{array}\right.
$$

It is easy to see that $\operatorname{Rot}_{\theta}$ is nonexpansive. Clearly, zero is the only fixed point of the mapping $\operatorname{Rot}_{\theta}$. Let $\gamma_{n}=1-\frac{1}{\sqrt{n+1}}$ and $\beta_{n}=\frac{1}{n+1}$ for all $n \in \mathbb{N}$ and without perturbations. By using Wolfram Mahtematica 10, we computed the iterates of (SM0) for an initial point $x_{(1)}=(250,250)$ for $\theta=\frac{\pi}{4}, \frac{\pi}{8}$ and $\frac{\pi}{14}$. The convergence behavior of all iterations for approximating the fixed point $(0,0)$ are given in Figure 1 and Table 1. 
FIGURE 2. Erro convergence behavior for Ex. 2.

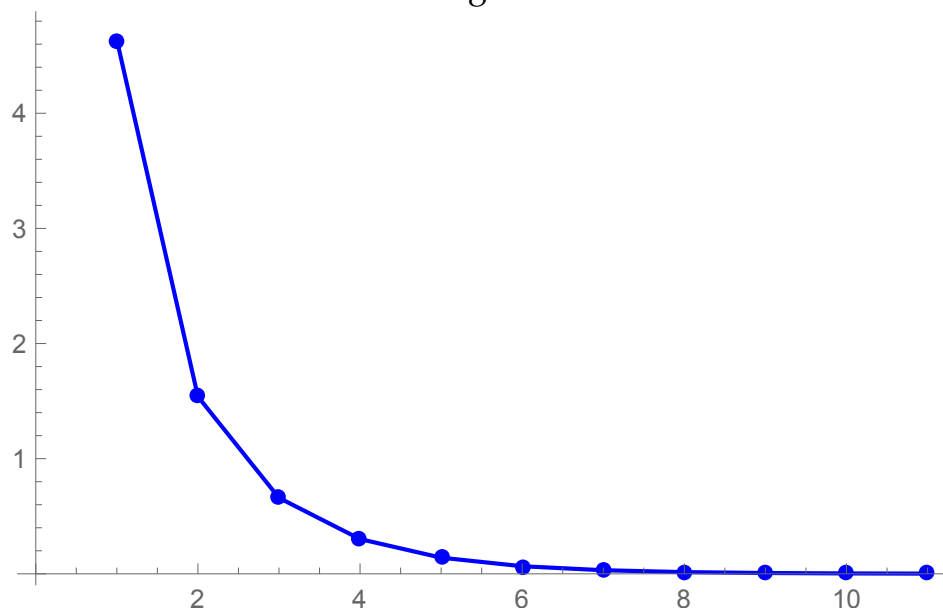

Example 4.2. Consider $X=\mathbb{R}^{3}$ equipped with the Euclidean norm. Define the nonexpansive mappings $T_{1}, T_{2}, T: X \rightarrow X$ as follows:

$$
T_{1}(x, y, z)=T_{2}(x, y, z)=T(x, y, z)=\left(\frac{1}{5}(3 x-2 y-8), \frac{1}{5}(-2 x+3 y-8), \frac{z}{2}\right) .
$$

We define $f: \mathbb{R}^{3} \rightarrow \mathbb{R}$ by

$$
f(u)=\frac{1}{2}\|A u-b\|^{2}, \quad u \in \mathbb{R}^{3},
$$

where

$$
A=\left(\begin{array}{ccc}
1 & 1 & -1 \\
1 & 1 & -1 \\
-1 & -1 & 1
\end{array}\right), \quad b=(-4,-4,4)
$$

The function $f$ is proper convex and lower semi-continuous. Hence for $\lambda>0$, we have

$$
J_{\lambda}(u)=\left(I+\lambda A^{*} A\right)^{-1}\left(u+\lambda A^{*} b\right) .
$$

Note that

$$
\Omega=F i x(T) \cap \operatorname{argmin}_{y \in X} f(y)=\left\{(x, y, z) \in \mathbb{R}^{3}: x+y+4=z=0\right\} .
$$

The algorithm (SM0) becomes:

$$
\left\{\begin{array}{l}
z_{n}=\left(I+\lambda_{n} A^{*} A\right)^{-1}\left(x_{n}+\lambda_{n} A^{*} b\right), \\
y_{n}=\left(1-\beta_{n}-\beta_{n}^{\prime}\right) x_{n} \oplus \beta_{n} T_{2} z_{n} \oplus \beta_{n}^{\prime} \varepsilon_{n}^{\prime}, \\
x_{n+1}=\left(1-\gamma_{n}-\gamma_{n}^{\prime}\right) T_{2} x_{n} \oplus \gamma_{n} T_{1} y_{n} \oplus \gamma_{n}^{\prime} \varepsilon_{n},
\end{array}\right.
$$

We choose $\lambda_{n}=0.35, \gamma_{n}^{\prime}=\beta_{n}^{\prime}=0 \gamma_{n}=1-\frac{1}{\sqrt{n+1}}$ and $\beta_{n}=\frac{1}{n+1}$ for all $n \in \mathbb{N}$. By using Wolfram Mahtematica 10, we computed the iterates of (SM0) for an initial point $x_{(1)}=(-1,2,3)$. The convergence behavior of all iterations for approximating the fixed point $(-3.5,-0.5,0)$ are given in Figure 2 and Table 2 . 
TABLE 2. Error convergence behavior for Ex. 2

\begin{tabular}{l|lll|l} 
Iteration & $x_{i}$ & $y_{i}$ & $z_{i}$ & Error \\
\hline 0 & -1. & 2. & 3. & 4.63681 \\
1 & -3.06154 & -0.0615429 & 1.40869 & 1.53912 \\
2 & -3.42143 & -0.421434 & 0.649992 & 0.65942 \\
3 & -3.48523 & -0.485235 & 0.300787 & 0.301511 \\
4 & -3.497 & -0.497005 & 0.140183 & 0.140247 \\
5 & -3.49931 & -0.499314 & 0.0657979 & 0.0658051 \\
6 & -3.49981 & -0.499815 & 0.0310751 & 0.0310762 \\
7 & -3.49994 & -0.49994 & 0.0147528 & 0.0147531 \\
8 & -3.49998 & -0.499978 & 0.00703466 & 0.00703473 \\
9 & -3.49999 & -0.499991 & 0.00336684 & 0.00336687 \\
10 & -3.5 & -0.499996 & 0.00161652 & 0.00161653 \\
$\vdots$ & $\vdots$ & $\vdots$ & $\vdots$ & $\vdots$ \\
20 & -3.5 & -0.5 & $1.173 \times 10^{-6}$ & $1.173 \times 10^{-6}$
\end{tabular}

\section{REFERENCES}

[1] Argarwal, R. P., OŔegan, D. and Sahu, D. R., Iterative construction of fixed points of nearly asymptotically nonexpansive mappings, J. Nonlinear Convex. Anal, 8 (2007), No. 1, 61-79

[2] Ariza Ruiz, D. A., Acedo, G. L. and Nicolae, A., The asymptotic behavior of the composition of firmly nonexpansive mappings, arXiv:1411.6779v1 [math.FA] 25 Nov. 2014

[3] Ariza-Ruiz, D., Leustean, L. and Lopez, G., Firmly nonexpansive mappings in classes of geodesic spaces, Trans. Am. Math. Soc., 366 (2014), 4299-4322

[4] Bachar, M. and Khamsi, M. A., Approximations of fixed points in the Hadamard metric space $C A T_{p}(0)$, Mathematics, 7 (2019), No. 11, 1088 https: / /doi.org/10.3390/math7111088

[5] Berinde, V., Iterative approximation of fixed points, Lecture Notes in Mathematics 1912, (2007)

[6] Bridson, M. and Haefliger, A., Metric spaces of non-positive curvature, Springer-Verlag, Berlin, Heidelberg, New York, 1999

[7] Calderon, K., Martinez-Moreno, J. and Rojas, E. M., Hybrid algorithm with perturbations for total asymptotically non-expansive mappings in CAT(0) space, Int. J. Comput. Math., 97 (2020), No. 1-2, 405-419

[8] Choi, B. J. and Ji, U. C., The proximal point algorithm in uniformly convex metric spaces, Commun. Korean Math. Soc., 31 (2016), 845-855

[9] Cholamjiak, P., The SP-iteration process for nonexpansive mappings in $C A T(\kappa)$ spaces, J. Nonlinear and Convex Anal., 16 (2015), No. 1, 109-118

[10] Cholamjiak, P., Abdou A. A. N. and Cho, Y. J., Proximal point algorithms involving fixed points of nonexpansive mappings in CAT(0) spaces, Fixed Point Theory Appl., 2015, 2015:227, 13 pp.

[11] Dhompongsa, S. and Panyanak, B., On $\Delta$-convergence theorems in CAT(0) spaces, Comput. Math. Appl., 56 (2008), 2572-2579

[12] Espínola, R. and Fernández-León, A., CAT(k)-spaces, weak convergence and fixed points, J. Math. Anal. Appl., 353 (2009), 410-427

[13] Goebel, K. and Japón Pineda, M., "A new type of nonexpansiveness", Proc. of the 8th International Conference on Fixed Point Theory and Appl., Chiang Mai, 2007

[14] Jost, J., Convex functionals and generalized harmonic maps into spaces of nonpositive curvature, Comment. Math. Helv., 70 (1995), 659-673

[15] Kimura, Y. and Kohsaka, F., Spherical nonspreadingness of resolvents of convex functions in geodesic spaces, J. Fixed Point Theory Appl., 18 (2016), 93-115

[16] Kimura, Y. and Kohsaka, F., The proximal algorithms in geodesic spaces with curvature bounded above, Linear and Nonlinear Anal., 3 (2017), 133-148

[17] Kirk, W. A., Geodesic geometry and fixed point theory, In: Seminar of Mathematical Analysis (Malaga/Seville, 2002/2003). Colecc. Abierta. Universidad de Sevilla Secretariado de Publicaciones, Sevilla, 64 (2003), 195-225

[18] Kirk, W. A., Geodesic geometry and fixed point theory II, In: International Conference on Fixed Point Theo. Appl., Yokohama Publ., Yokohama, (2004), 113-142 
[19] Kirk, W.A. and Panyanak, B., A concept of convergence in geodesic spaces, Nonlinear Anal., 68 (2008), 3689-3696

[20] Khamsi, M. A. and Shukri, S. A., Generalized CAT(0) spaces, Bull. Belg. Math. Soc. Simon Stevin, 24 (2017), No. 3, 417-426

[21] Klin-eam, C. and Suantai, S., "Fixed point theorems for alpha-nonexpansive mappings", Appl. Math. Lett., 23 (2010), 728-731

[22] Kuwae K., Resolvent ows for convex functionals and p-Harmonic maps, Anal. Geom. Metr. Spaces, 3 (2015), $46-72$

[23] Lim, T. C., Fixed point theorem for uniformly Lipschitzian mappings in Lp spaces, Nonlinear Anal., 7 (1983), $555-563$

[24] Liu, L. S., Ishikawa and Mann Iterative Process with Errors for Nonlinear Strongly Accretive Mappings in Banach Spaces, J. Math. Anal. Appl., 194 (1995), No. 1, 114-125

[25] Mayer, U. F., Gradient flows on nonpositively curved metric spaces and harmonic maps, Comm. Anal. Geom., 6 (1998), No. 2, 199-253

[26] Moreau J. J., Fonctions convexes duales et points proximaux dans un espace hilbertien, C. R. Acad. Sci. Paris, 255 (1962), 2897-2899

[27] Naor, A. and Silberman, L., Poincaré inequalities, embeddings, and wild groups, Compos. Math., 147 (2011), No. $5,1546-1572$

[28] Ohta, S.-I., Convexities of metric spaces, Geom. Dedicata, 125 (2007), 225-250

[29] Pakkaranang, N., Kumam, P., Cholamjiak, P., Suparatulatorn, R. and Chaipunya P., Proximal point algorithms involving fixed point iteration for nonexpansive mappings in $C A T(\kappa)$ spaces, Carpathian J. Math., 34 (2018), No. 2, 229-237

[30] Pakkaranang, N., Kumam, P., Wen, C. F., Yao, J. C. and Cho, Y. J., On modified proximal point algorithms for solving minimization problems and fixed point problems in $C A T(\kappa)$ spaces, To apper in Mathematical Methods in the Applied Sciences.

[31] Qihou, L., Convergence theorems of the sequence of iterates for asymptotically demicontractive and hemicontractive mappings, Nonlinear Anal., 26 (1996), 1835-1842

[32] Xu, Z-B. and Roach, G. F., Characteristic Inequalities of Uniformly Convex and Uniformly Smooth Banach Spaces, J. Math. Anal. Appl., 157 (1991), 189-210

[33] Xu, H. K., Inequalities in Banach spaces with applications, Nonlinear Analysis, 16 (1991), No. 12, 1127-1138

[34] Xu, Y., Ishikawa and Mann Iterative Processes with Errors for Nonlinear Strongly Accretive Operator Equations, J. Math. Anal. Appl., 224 (1998)., No. 1, 91-101

\author{
${ }^{1}$ UnIVERSIDAD DE CienCIAS APLiCADAS y AMBIENTALES \\ FACULTAD DE CiENCIAS \\ BOGOTÁ, COLOMBIA \\ Email address: kencalderon@udca.edu.co \\ ${ }^{2}$ Department of Mathematics \\ KHALIFA UNIVERSITY \\ ABU DHABIA, UAE \\ Email address: mohamed.khamsi@ku.ac.ae \\ ${ }^{3}$ Department of Mathematics \\ UNIVERSIDAD DE JAÉN \\ JAÉN, SPAIN \\ Email address: jmmoreno@ujaen.es
}

